\title{
Review \\ Strategies to optimize analgesia and sedation
} William D Schweickert ${ }^{1}$ and John $\mathrm{P}$ Kress ${ }^{2}$

\begin{abstract}
1Division of Pulmonary, Allergy and Critical Care, University of Pennsylvania Medical Center, Spruce Street, Philadelphia, Pennsylvania 19104, USA ${ }^{2}$ Section of Pulmonary and Critical Care, University of Chicago Hospitals, Maryland Avenue, Chicago, Illinois 60637, USA
\end{abstract}

Corresponding author: John P Kress, jkress@medicine.bsd.uchicago.edu

Published: 14 May 2008

This article is online at http://ccforum.com/content/12/S3/S6

(c) 2008 BioMed Central Ltd
Critical Care 2008, 12(Suppl 3):S6 (doi:10.1186/cc6151)

tions, but a need for sedatives and analgesics to promote tolerance to the intensive care unit (ICU) environment is typically the rule.

Sedation needs vary widely in mechanically ventilated patients. ICU patients frequently exhibit unpredictable pharmacology with accumulation of drug in tissue stores, resulting in a prolonged clinical effect. Other variables that confound attempts to predict drug effect include renal and hepatic dysfunction, drug-drug interactions, hypoproteinemia, and shock. As a result, sedatives and analgesics must be titrated to discernible and reproducible clinical end-points. Because the drugs used in this context are extremely potent, clinicians must have heightened awareness of the potential for enduring effects and are encouraged to employ strategies that maximize benefit while minimizing risk.

The risk for untreated pain or agitation is a primary concern. Most mechanically ventilated patients experience some degree of pain even in the absence of surgical incisions or trauma $[1,2]$. Accordingly, it is critical for clinicians to direct initial attention toward analgesia when they administer 'sedation'. Untreated pain may cause many adverse effects, including increased endogenous catecholamine activity, myocardial ischemia, hypercoagulability, hypermetabolic states, sleep deprivation, anxiety, and delirium [3]; treating this pain has been shown to ameliorate some of these effects [4]. Untreated agitation, particularly in the delirious patient, may result in similar problems, including patient self-injury via removal of life-sustaining devices.

In contrast, some patients may exhibit extreme hypertension, tachycardia, tachypnea, and ventilator asynchrony even in a comatose state. These findings are particularly common in unconventional ventilation strategies (low tidal volumes, permissive hypercapnea, high-frequency oscillatory ventilation, and prone positioning) and may counter the body's usual

$\mathrm{AOD}=$ alcohol and other drug use disorders; ATICE = Adaptation to the Intensive Care Unit Environment; DIS = daily interruption of sedative infusions; ICU = intensive care unit; MICU = medical intensive care unit; PTSD = post-traumatic stress disorder. 


\section{Figure 1}

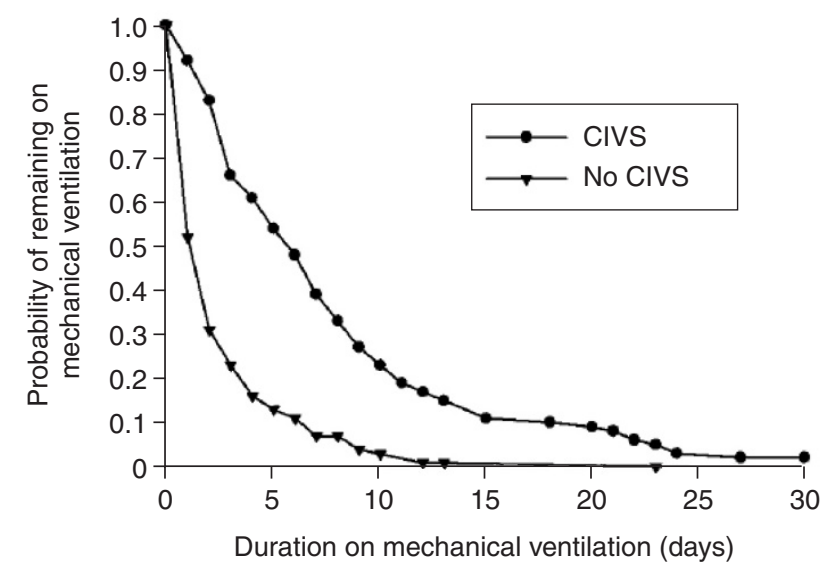

Duration of mechanical ventilation: continuous intravenous sedation versus interrupted/no intravenous sedation. Shown are Kaplan-Meier curves for patients receiving continuous intravenous sedation (CIVS) and patients not receiving continuous intravenous sedation. Reprinted with permission from Chest 1998, 114:541-548. (C) American College of Chest Physicians.

response to stress. With increasing acuity of illness, it may become difficult to reconcile the goals of ensuring patient tranquility while preventing accumulation of sedatives and analgesics.

The resulting deep sedation may have significant unintended consequences. Kollef and coworkers [5] studied the outcomes of 240 mechanically ventilated patients stratified by sedation strategy, namely continuous intravenous sedation or interrupted/no intravenous sedation. Patients undergoing continuous intravenous sedation had markedly increased duration of mechanical ventilation, ICU and hospital length of stay, organ system failure, and re-intubation rates (Figure 1). Although the study is limited by the lack of randomization, the difference in duration of mechanical ventilation persisted after multivariate analysis incorporating severity of illness, mortality, indication for mechanical ventilation, use of chemical paralysis, presence of tracheostomy, and number of acquired organ system derangements.

Additionally, investigators are now measuring other deleterious outcomes that may (at least in part) stem from sedation. Central nervous system dysfunction, manifested as delirium, occurs in $60 \%$ to $80 \%$ of patients undergoing mechanical ventilation [6-8]. ICU delirium is predictive of a threefold higher re-intubation rate and more than 10 additional days in the hospital, and it is independently associated with higher ICU, inpatient, and 6-month mortality rates [9-12]. An evaluation of sedative and analgesic use in ICU patients [13] demonstrated a dose-related association between lorazepam administration and the daily transition to delirium. Studies are now ongoing to identify a cause-effect relationship in order to evaluate whether serial assessments for delirium will promote improved sedation behaviors and patient outcomes.

The ideal sedative and analgesic regimen will provide adequate sedation and pain control, be of rapid onset of action and allow rapid recovery after discontinuation, have minimal systemic accumulation, and carry minimal adverse effects, without increasing overall health care costs (which is a challenge in the development of a novel drug or application). Candidate drugs include ultra-short-acting agents such as remifentanil and another class of agent (other than propofol, benzodiazepines, and opioids) such as the $\alpha_{2}$ adrenergic agonist dexmedetomidine. These drugs (discussed in detail in other reviews included in this supplement $[14,15]$ ) herald great promise in longer term analgesia and sedation strategies. Further studies of these drugs' influence on outcomes such as duration of mechanical ventilation, length of ICU stay, mortality, and delirium will help to establish their role in routine practice.

Until that time, reliance upon short-acting (propofol) and intermediate- and long-acting (benzodiazepines and opioids) agents is common practice, and recent clinical trials now permit an evidence-based approach to analgesia and sedation management in the ICU. To understand how sedation protocols should function optimally, we review the evidence and practical implementation of researched strategies.

\section{Patient-targeted sedation protocols}

Patient-targeted sedation protocols implement two main features: a structured approach to the assessment of patient pain and distress, coupled with an algorithm that directs drug escalation and de-escalation based on the assessments.

Limited data suggest that routine, structured assessments alone may effect substantial change on outcomes. Chanques and coworkers [16] studied the frequency of pain and agitation in ICU patients in a period preceding and following the incorporation of analgesia and sedation scales. In the initial phase, no systematic and objective evaluation of pain or agitation was performed by nurses or physicians. During a brief interphase, nurses and physicians were educated on the application of the Behavioral Pain Scale [17], Numerical Rating Scale for pain [18], and the Richmond Agitation Sedation Scale [19]. During the intervention phase, critically ill patients experienced less pain and agitation (although assessments were not conducted by independent observers), more frequent titrations of both analgesic and psychoactive drugs were performed, and duration of mechanical ventilation was reduced.

More robust data exist documenting the success of protocols that link assessments to consequent actions. Patient-targeted sedation protocols have been tested in multiple investigations conducted in the setting of single-center, two-phase, prospective, controlled studies, with varying degrees of 


\begin{tabular}{|c|c|c|c|c|c|c|c|c|c|}
\hline \multicolumn{4}{|c|}{ Consciousness domain } & \multicolumn{6}{|c|}{ Tolerance domain } \\
\hline \multicolumn{2}{|l|}{$\begin{array}{l}\text { AWAKENESS } \\
\text { (graded } 0 \text { to } 5 \text { ) }\end{array}$} & \multicolumn{2}{|c|}{$\begin{array}{l}\text { COMPREHENSION } \\
\text { (sum of the } \\
\text { 1-point responses) }\end{array}$} & \multicolumn{2}{|l|}{$\begin{array}{l}\text { CALMNESS } \\
\text { (graded } 0 \text { to } 3 \text { ) }\end{array}$} & \multicolumn{2}{|c|}{$\begin{array}{c}\text { VENTILATOR SYNCHRONY } \\
\text { (sum of the } \\
\text { 1-point elements) }\end{array}$} & \multicolumn{2}{|c|}{$\begin{array}{l}\text { FACE RELAXATION } \\
\text { (graded } 0 \text { to } 3 \text { ) }\end{array}$} \\
\hline eyes closed, no mimic & 0 & $\begin{array}{l}\text { «open (close) your } \\
\text { eyes » }\end{array}$ & 1 & $\begin{array}{l}\text { life-threatening } \\
\text { agitation }\end{array}$ & 0 & $\begin{array}{l}\text { no blockade of the } \\
\text { inspiratory phase of } \\
\text { ventilation }\end{array}$ & 1 & $\begin{array}{l}\text { permanent } \\
\text { grimacing }\end{array}$ & 0 \\
\hline $\begin{array}{l}\text { eyes closed, only face } \\
\text { mimic after strong } \\
\text { painful stimulation }\end{array}$ & 1 & « open your mouth » & 1 & & & & \multirow[b]{2}{*}{1} & & \\
\hline $\begin{array}{l}\text { eyes opening after } \\
\text { strong painful } \\
\text { stimulation }\end{array}$ & 2 & «look at me» & 1 & $\begin{array}{l}\text { agitation, does not } \\
\text { respond to verbal } \\
\text { order }\end{array}$ & 1 & $\begin{array}{l}\text { no inspiratory rate } \\
>30\end{array}$ & & $\begin{array}{l}\text { severe provoked } \\
\text { grimacing }\end{array}$ & 1 \\
\hline $\begin{array}{l}\text { eyes opening after } \\
\text { light painful } \\
\text { stimulation }\end{array}$ & 3 & & & \multirow{2}{*}{$\begin{array}{l}\text { agitation, responds } \\
\text { to verbal order }\end{array}$} & \multirow[t]{2}{*}{2} & \multirow[t]{2}{*}{ no cough } & \multirow[t]{2}{*}{1} & \multirow{2}{*}{$\begin{array}{l}\text { moderate provoked } \\
\text { grimacing }\end{array}$} & \multirow[t]{2}{*}{2} \\
\hline eyes opening after & 4 & $\begin{array}{l}\text { « nod yes with } \\
\text { your head » }\end{array}$ & 1 & & & & & & \\
\hline $\begin{array}{l}\text { eyes opening } \\
\text { spontaneously }\end{array}$ & 5 & $\begin{array}{l}\text { « close your eyes } \\
\text { and open your } \\
\text { mouth » }\end{array}$ & 1 & calm & 3 & $\begin{array}{l}\text { no use of accessory } \\
\text { respiratory muscles }\end{array}$ & 1 & relaxed face & 3 \\
\hline
\end{tabular}

ATICE instrument. ATICE, Adaptation to the Intensive Care Unit Environment. Adapted with permission from De Jonghe B, Cook D, Griffith L, Appere-de-Vecchi C, Guyatt G, Theron V, Vagnerre A, Outin H: Adaptation to the Intensive Care Environment (ATICE): development and validation of a new sedation assessment instrument. Crit Care Med 2003, 31(9):2344-2354.

success [20-23]. However, the most compelling data supporting patient-targeted protocols comes from a randomized, controlled clinical trial of 321 patients receiving mechanical ventilation conducted in an urban teaching hospital's medical ICU (MICU). Brook and coworkers [24] compared the practice of protocol-directed sedation during mechanical ventilation implemented by nurses versus traditional non-protocol-directed administration of sedation. In patients randomly assigned to receive the protocol-directed sedation, nurses determined whether sedatives or analgesics, or both, were needed to provide optimal patient care. The type of sedation administration (intravenous bolus administration versus continuous intravenous infusion) and the dosage were determined by nursing staff, targeting an ideal level of sedation. In contrast, treating physicians managed all aspects of sedation in the non-protocol-directed group. In this latter group, nurses were able to communicate their opinions and observations, but they could not make changes without a physician's order. Although protocol deviation was allowed, the majority of patients were treated with benzodiazepines for sedation and fentanyl or morphine for analgesia.

The application of protocol-directed sedation resulted in a significantly shorter duration of mechanical ventilation (median duration 55.9 hours) compared with those receiving nonprotocol-directed sedation (117.0 hours) [24]. Additionally, ICU and hospital lengths of stay were both significantly shorter among patients in the protocol-directed sedation group. Approximately $40 \%$ of patients in each group underwent continuous intravenous sedation, but those in the protocol-directed sedation group had a significantly shorter duration of continuous infusion ( 3.5 days versus 5.6 days; $P=0.003)$. Patients in the protocol-directed sedation group also had a significantly lower tracheostomy rate than did those in the non-protocol-directed sedation group.

In practice, this protocol emphasized the importance of an initial assessment of analgesic needs followed by an assessment for agitation. Utilizing a combination of benzodiazepine and opioids, nurses sought to achieve an ideal level of sedation, defined as a score of 3 on the Ramsay Sedation Scale (patient responds to commands only) [25]. If frequent administrations of extra boluses of sedative or opioid were necessary, then continuous infusions were begun. Reassessment occurred at least every 4 hours, and downward titration of infusion rates was targeted until the infusion was stopped.

Most recently, De Jonghe and coworkjers [20] demonstrated that similar success can be achieved with the implementation of a sedation protocol that utilizes a more comprehensive patient assessment tool. To date, the majority of sedation protocols have relied upon the Ramsay Sedation Scale score, but this scale has attracted criticism because of its lack of clear discrimination and specific descriptors to differentiate between the various levels [26,27]. Furthermore, attention to agitation and ventilator synchrony is absent. In contrast, the Adaptation to the Intensive Care Unit Environment (ATICE) tool consists of five items (Figure 2) [28]: calmness, ventilator 


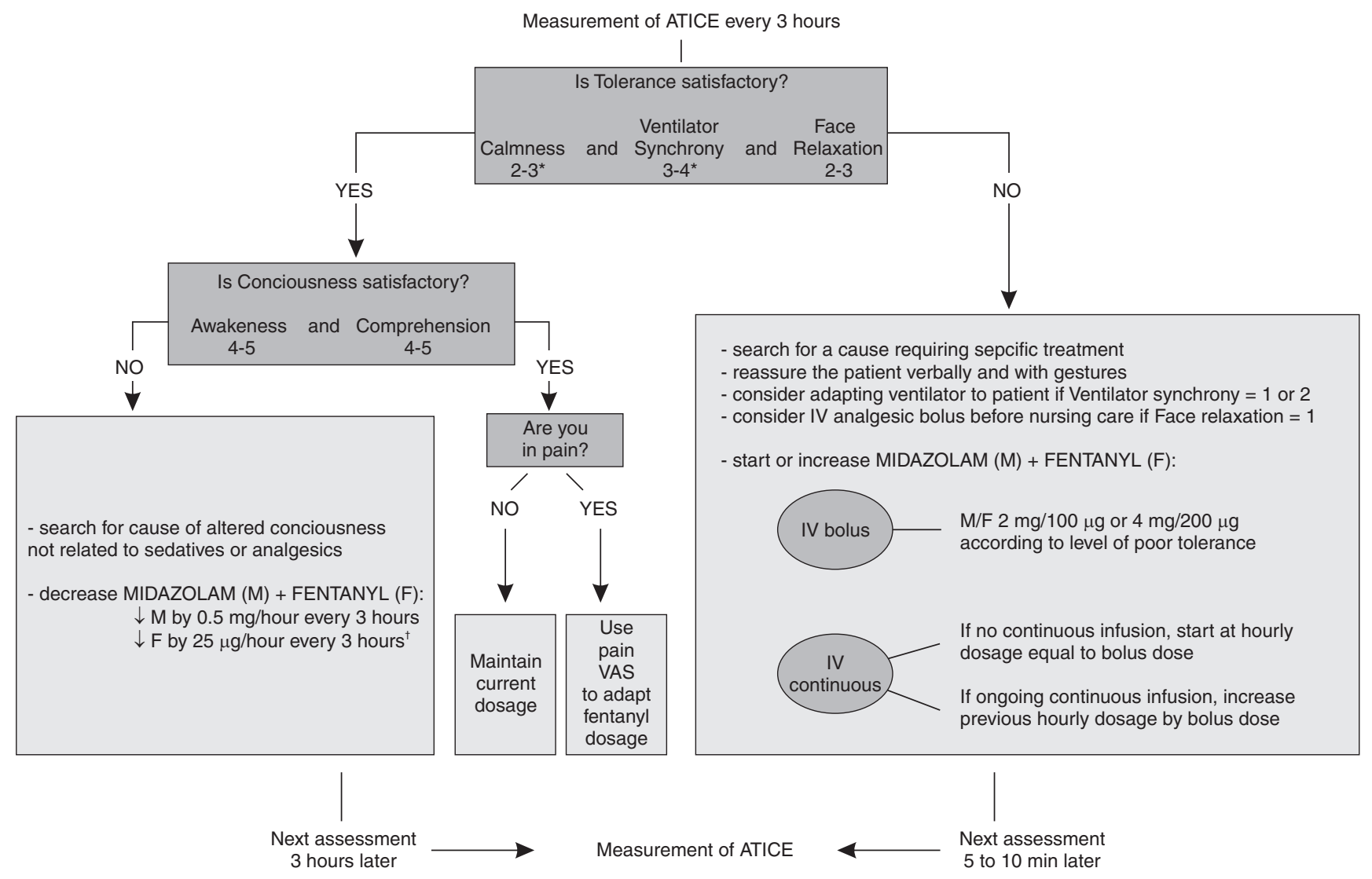

ATICE-based Sedation and Analgesia Algorithm. Adaptation to the Intensive Care Unit Environment (ATICE) is measured every 3 hours, except during the night in patients with satisfactory consciousness at the end of the day. ATICE is measured more frequently in patients with worsening tolerance. The algorithm is not applied while patients are receiving neuromuscular blockers, in the prone position, or after an end-of-life decision has been made. *Stricter objectives of tolerance (calmness $=3$, ventilator synchrony $=4$ ) may transiently be used in patients with severe acute respiratory or circulatory failure. ${ }^{\dagger}$ During the initial 48 -hour postoperative period, do not decrease fentanyl under 50 to $100 \mu \mathrm{g} / \mathrm{hour} . \downarrow$, decrease drug dosage; IV, intravenous; VAS, visual-analog scale. Reprinted with permission from De Jonghe B, Bastuji-Garin S, Fangio P, Lacherade JC, Jabot J, Appere-De-Vecchi C, Rocha N, Outin H: Sedation algorithm in critically ill patients without acute brain injury. Crit Care Med 2005, 33(1):120-127.

synchrony, and face relaxation combined in a 'tolerance' domain, and awakeness and comprehension combined in a 'consciousness' domain. Prior work had demonstrated that the scale, developed by a multidisciplinary team of nurses and physicians, exhibited high internal consistency, inter-rater reliability across disciplines, and validity. This instrument correlates well both with several published sedation scales and with amounts of sedatives and analgesics given to patients.

In the study conducted by De Jonghe and coworkers [20], the ATICE assessment was paired with a sedation algorithm that initially evaluated patients' 'tolerance' to the ICU environment (measuring calmness, ventilator synchrony, and facial expression; Figure 3). Derangements in any one of the categories prompted further sedative or opioid administration (assuming verbal reassurance and ventilator manipulation were ineffective). Patients with tolerance were then assessed for 'consciousness', in which sedatives and opioids were titrated down to achieve an interactive patient who could reply to commands and queries regarding the presence of pain.

Outcomes in 102 mechanically ventilated patients without acute brain injury were measured during two 6-month time periods [20]: preceding and following the implementation of the ATICE-based sedation protocol. Median duration of mechanical ventilation was significantly shorter in the algorithm group. This difference persisted with multivariate analysis that included factors such as severity of illness and organ dysfunction. Most importantly, the median time to arousal was also significantly shorter in patients in the algorithm group ( 2 days) than in the control group ( 4 days; $P=0.006$ ). This documented shortening of time to arousal is considered to be the most potent mechanism whereby sedation protocols can reduce the duration of mechanical ventilation. 
Analgesic and sedative administration (irrespective of route and type) guided to ensure patient safety and designed to promote tolerance to an uncomfortable environment and altered state of health can result in improved outcomes. Seemingly, this result stems from a more rapid return to an awake state, but other factors may be at play, including avoidance of protracted immobility, ileus, delirium, and dangerous agitation. Studies to explore these mechanisms may help to guide the next generation of drug selection and administration strategies.

\section{Daily interruption of sedative infusions}

If the primary goal is to achieve the earliest awakening possible, then an alternative sedation protocol strategy that may be applied is daily interruption of sedative infusions (DIS). This strategy employs a similar goal of sedative and analgesic titration to an optimal depth of sedation dictated by physicians or nursing staff, or both. In contrast to 'patienttargeted sedation protocols', no formal algorithm has been established for drug escalation. However, the risk for excessive sedation is minimized by a daily interruption of both sedative and analgesic infusions until the patient awakens or exhibits distress that mandates resumed drug administration.

This strategy was first introduced via a randomized controlled trial involving 128 adult patients who were receiving mechanical ventilation and continuous infusions of sedative drugs in an academic MICU [29]. The study, designed in a factorial $2 \times 2$ design, randomly assigned patients by sedation strategy: an intervention group, in which sedative infusions were interrupted on a daily basis until the patients were awake; and a control group, in which the infusions were interrupted only at the discretion of the clinicians in the ICU. The secondary randomization was based upon the sedative employed, namely propofol or midazolam (treatment in both groups was coupled with morphine for analgesia).

When the patients were evaluated by sedation strategy, the median duration of mechanical ventilation was 4.9 days in the intervention group versus 7.3 days in the control group $(P=0.004)$, and the median lengths of stay in the ICU were 6.4 days and 9.9 days, respectively ( $P=0.02$; Figure 4) [29]. The DIS approach conferred additional advantages, including a marked difference in the number of days during which patients were awake and following commands $(85.5 \%$ versus 9.0\%; $P<0.001)$. This culminated in a significant reduction in the need for diagnostic testing (via imaging or lumbar puncture) to assess changes in mental status ( $9 \%$ of patients versus $27 \% ; P=0.02)$. Complications related to undersedation (for instance, removal of the endotracheal tube by the patient) were no different between groups ( $4 \%$ in the intervention group versus $7 \%$ in the control group; $P=0.88$ ).

Evaluation of groups based on sedative employed demonstrated no difference between propofol and midazolam with respect to the study's primary end-points: duration of mechanical ventilation, and ICU and hospital lengths of stay. Of note, both total dosage and average rate of infusion for both midazolam and morphine were reduced by half in the intervention group (DIS). This reflects the ability of the DIS approach to minimize drug accumulation and avoid delayed drug emergence and so shorten the duration of mechanical ventilation (akin to patient-targeted sedation protocols). However, the protocol was equally effective for patients randomly assigned to propofol infusion, prompting further questions about possible unrecognized benefits that accrue from complete interruption (reduced need for vasoactive drug, less fluid administration, and spontaneous muscle movement). Although the mechanism of benefit has not been fully evaluated, the improved outcomes are compelling.

The practice of DIS generates more complex discussion than patient-titrated sedation protocols. Both sedative and analgesic agents should be interrupted once daily, unless there is evidence for ongoing patient distress, reasonable certainty that there is ongoing pain, or utilization of neuromuscular blockade. Once the drugs are interrupted, the ICU team must be vigilant for evidence of patient distress, which may manifest as overt physical agitation, isolated hemodynamic lability (hypertension or tachycardia), or ventilator asynchrony. Providers are then encouraged to administer bolus drug dosing to de-escalate symptoms, and restart both sedative and analgesic drugs at half the previous infusion doses with subsequent titration to the desired depth of sedation.

The study met with criticism because of its single center application, absence of a standardized approach to ventilator weaning, and, most markedly, concerns for potentially unrecognized patient harm from the daily interruption [30]. These concerns include fear of precipitating psychologic distress, myocardial ischemia, or drug and alcohol withdrawal syndromes.

Long-term consequences of recovery from respiratory failure include depression, anxiety, and post-traumatic stress disorder (PTSD). Limited data suggest a positive relationship between duration of sedation and development of depression and PTSD [31]. Although it had traditionally been viewed that unpleasant memories of critical illness may elicit psychiatric illness, newer data suggest that the lack of recall of factual events in the ICU is associated with a heightened risk for PTSD in survivors [32]. Therefore, DIS creates a conundrum; it may potentially be protective as a means to achieve awakening with an increased likelihood of patient recall, or it may risk further psychologic distress resulting from the nature of the abrupt changes in level of consciousness.

To address this uncertain outcome, a cohort of survivors of critical illness requiring mechanical ventilation and sedation underwent psychologic assessment with outcomes stratified by the sedation strategy [33]. Psychologists blinded to patient randomization assessed for signs of PTSD, anxiety, 


\section{Figure 4}

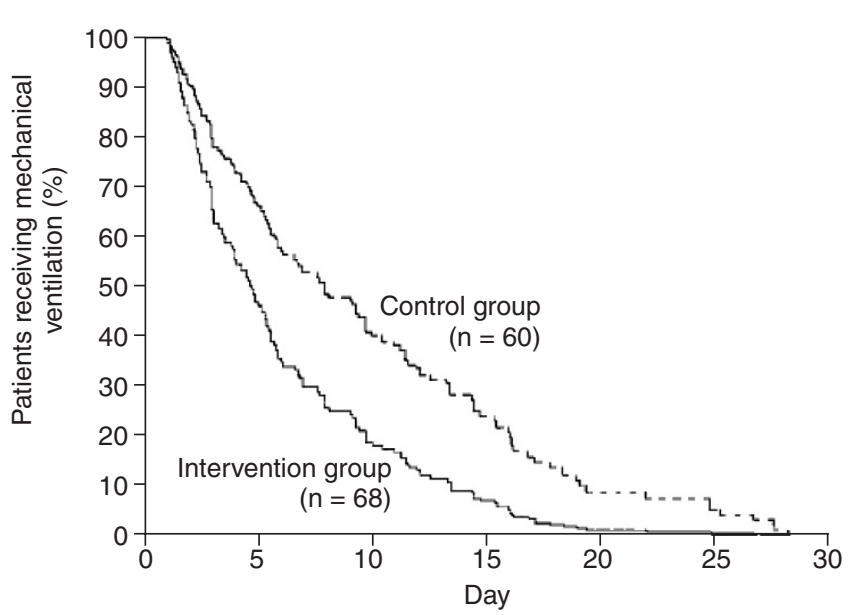

Duration of mechanical ventilation: DIS versus control. Shown is a Kaplan-Meier analysis of the duration of mechanical ventilation, according to study group: an intervention group, in which sedative infusions were interrupted on a daily basis (DIS) until the patients were awake; and a control group, in which the infusions were interrupted only at the discretion of the clinicians in the intensive care unit. After adjustment for baseline variables (age, sex, weight, Acute Physiology and Chronic Health Evaluation II score, and type of respiratory failure), mechanical ventilation was discontinued earlier in the intervention group than in the control group (relative risk for extubation $=1.9,95 \%$ confidence interval $=1.3$ to $2.7 ; P<0.001$ ). Reprinted with permission from Kress JP, Pohlman AS, O'Connor MF, Hall JB: Daily interruption of sedative infusions in critically ill patients undergoing mechanical ventilation. N Engl J Med 2000, 342(20):1471-1477. Copyright (C) 2000 Massachusetts Medical Society. All rights reserved.

depression, and psychosocial adjustment to illness. The cohort of patients who experienced DIS (versus continuous sedative infusion) had a better total Impact of Events score [34] (representing less frequent PTSD signs; 11.2 versus 27.3; $P=0.02)$, a trend toward a lower incidence of PTSD [35] (0\% versus 32\%; $P=0.06)$, and a trend toward a better total Psychosocial Adjustment to Illness score [36] (46.8 versus 54.3; $P=0.08$ ). Patients undergoing DIS had shorter durations of mechanical ventilation; consequently, it is not possible to confirm that the sedation strategy may be the only factor that contributes to the psychologic outcome. However, the study does demonstrate that DIS did not lead to harmful outcomes in this cohort.

The second concern, the possibility that DIS may precipitate myocardial ischemia, is understandable given the established recognition of ischemia with procedures such as intubation and spontaneous breathing trials [37-41]. In response, we evaluated the prevalence of myocardial ischemia in 74 mechanically ventilated patients with coronary risk factors, comparing periods of sedative interruption with sedative infusion [42]. Ischemia, defined by ST-segment changes documented by continuous three-lead Holter monitor, was interpreted by a cardiologist who was blinded to sedation status. In the cohort of 74 patients, $24 \%$ exhibited ischemic changes during the period of mechanical ventilation. Periods of awakening from sedation (versus periods of sedative infusion) were associated with significantly increased heart rate, mean arterial pressure, rate-pressure product, respiratory rate, and catecholamine levels. Despite these physiologic changes during sedative interruption, the fraction of ischemic time did not differ between the time awake and the time sedated. Therefore, within the limits of this study design, myocardial ischemia was found to occur commonly during mechanical ventilation in critically ill patients with coronary artery disease risk factors, but DIS was not associated with a heightened risk for ischemia.

Finally, concern for the interplay between sedative interruption and withdrawal syndromes is understandable. Alcohol and other drug use disorders (AOD) affect 9.4\% of the American population [43], and the prevalence of these disorders in ICUs ranges from 9\% to 39\% [44-48]. Studies have demonstrated that selected patients with AOD have a greater likelihood of being admitted to an ICU, increased risks for requiring mechanical ventilation and developing sepsis, septic shock, and acute lung injury, and increased hospital mortality $[47,49-52]$. In a single center, retrospective review of 70 mechanically ventilated patients, De Wit and coworkers [48] documented AOD in 39\% of patients. This cohort experienced a 2.5-fold increased requirement for sedatives and a fivefold higher dose of opioids compared with patients without these disorders, but similar levels of sedation and frequency of agitation were documented. Balancing of this need for high doses of sedative and analgesic drugs in mechanically ventilated patients with AOD against a desire to minimize accumulation sets the stage for evolution of dangerous withdrawal syndromes. Further investigation into the safety of DIS in this population is needed.

\section{Continuous versus intermittent sedative administration}

Given concern that continuous sedative infusions carry such heightened risk for excessive sedation, guidelines have postulated that intermittent bolus techniques utilizing benzodiazepines might be an equivalent (or superior) sedation strategy, even with daily interruption of sedative infusions [53]. The comparison of these two strategies was studied in two academic centers' MICUs in an open label trial of 132 patients requiring more than 48 hours of mechanical ventilation and moderate to high levels of sedation [54]. Patients were randomly assigned to receive lorazepam by intermittent bolus administration or continuous infusions of propofol. In both groups, patients had sedation titrated to achieve a target Ramsay Sedation Scale score of 2 (patient cooperative, oriented, and tranquil) to 3 (patient responds to commands only) [25], assessed every 2 hours. In the intermittent group lorazepam was administered with usual dose ranges of 2 to $4 \mathrm{mg}$ every 4 hours; a peak dose of $8 \mathrm{mg}$ was considered acceptable. The continuous infusion group had 
propofol titrated to peak dose of $4.8 \mathrm{mg} / \mathrm{kg}$ per hour $(80 \mu \mathrm{g} / \mathrm{kg}$ per minute). Both groups were administered concomitant analgesia (utilizing morphine with allowance for continuous drips if ventilator asynchrony was present) and daily interruption of sedation and analgesics. Patients were deemed treatment failures if adequate sedation was not achieved by $8 \mathrm{mg}$ lorazepam every 4 hours or a maximum dosage of $4.8 \mathrm{mg} / \mathrm{kg}$ per hour propofol, or - in the case of propofol development of hypertriglyceridemia, bradycardia, or hypotension not attributable to another cause.

Although both groups had parallel distributions of time spent in different levels of sedation, the primary outcome, namely median ventilator days, was significantly lower in the daily interruption propofol group than in the intermittent bolus lorazepam group (5.8 days versus 8.4 days; $P=0.04$; Table 1). The difference was greatest for hospital survivors (4.4 days versus 9.0 days; $P=0.006$ ). Treatment failures were low in both groups ( $4 \%$ and $8 \%$ ). Of note, rates of selfextubation were low in both groups $(<5 \%)$, further supporting the safety of daily interruption of sedation.

The mechanism of benefit with daily interruption of continuous propofol infusions cannot be delineated from the available data. Possible explanations include persistent sedative effect of benzodiazepines impairing spontaneous breathing trial performance or increasing rates of delirium. Alternatively, patients in the propofol arm did receive higher morphine doses, which might have helped to improve the frequency to tidal volume ratio observed during the spontaneous breathing trials. Regardless, the randomized study design, coupled with its multicenter implementation, further strengthen the support for DIS, particularly with protocols employing propofol. However, DIS has not been tested utilizing ultra-short-acting agents such as remifentanil, for which downward titration may be more appropriate until data are available.

\section{Implementing a sedation protocol}

Despite the success of sedation protocols outlined above, there is still surprisingly low implementation of sedation scoring systems and sedation protocols in general practice. Recent surveys of sedation practice patterns in Canada [55], the USA [56], and Denmark [57] documented that formalized sedation scoring systems/assessment tools are present in $\leq 50 \%$ of critical care units, with sedation protocols being utilized in $\leq 33 \%$.

Some of the reluctance to adopt sedation protocols may stem from the absence of large-scale, multicenter, randomized trials in the field, and from institutional and individual bias regarding sedation scales and agents employed. However, we believe that existing data support certain conclusions. Successful sedation protocol implementation requires three factors: frequent assessment of sedation and analgesia using a reproducible scale; combination therapy
Table 1

Outcomes between patients randomized to intermittent bolus lorazepam versus propofol with daily interruption

\begin{tabular}{|c|c|c|c|}
\hline & Lorazepam & Propofol & $P$ \\
\hline \multicolumn{4}{|l|}{ Ventilator days } \\
\hline All patients & $8.4(4.6,14.7)$ & $5.8(3.5,10.3)$ & 0.04 \\
\hline Survivors & $9.0(5.3,16.8)$ & $4.4(3.0,8.7)$ & 0.006 \\
\hline Nonsurvivors & $7.5(4.0,11.4)$ & $7.2(4.2,13.2)$ & 0.66 \\
\hline \multicolumn{4}{|c|}{ 28-day ventilator-free survival (days) } \\
\hline All patients & $10.2(0,20)$ & $18.5(0,24)$ & 0.06 \\
\hline \multicolumn{4}{|l|}{ ICU length of stay (days) } \\
\hline All patients & $10.4(6.7,16.8)$ & $8.3(5.2,15.2)$ & 0.20 \\
\hline Survivors & $12.7(7.8,19.1)$ & $8.6(5.0,14.7)$ & 0.05 \\
\hline Nonsurvivors & $9.5(5.3,12.0)$ & $9.3(6.0,18.1)$ & 0.68 \\
\hline \multicolumn{4}{|c|}{ Hospital length of stay (days) } \\
\hline All patients & $20(12,30)$ & $18(12,29)$ & 0.55 \\
\hline Survivors & $22.5(14,33)$ & $19(10,32)$ & 0.16 \\
\hline Hospital mortality (n [\%]) & $24(38)$ & $25(37)$ & 0.82 \\
\hline
\end{tabular}

The total numbers of patients were 64 for the lorazepam group and 68 for the propofol group. Respectively, 40 and 43 patients survived ('survivors'), and 24 and 25 patients did not survive ('nonsurvivors'). Unless otherwise stated, values are expressed as median (25th, 75th percentiles). ICU, intensive care unit. Adapted with permission from Carson SS, Kress JP, Rodgers JE, Vinayak A, Campbell-Bright S, Levitt J, Bourdet S, Ivanova A, Henderson AG, Pohlman A, et al:: A randomized trial of intermittent lorazepam versus propofol with daily interruption in mechanically ventilated patients. Crit Care Med 2006, 34(5):1326-1332.

coupling sedatives and opioids with dosing adjustments guided by the scale; and, most importantly, careful communication between team members, with particular recognition that the bedside nurse must be empowered to pair assessments with drug manipulation (Figure 5).

Critical care unit leadership must choose among an expansive choice of sedation scales employed for patient assessment. Sedation scales (discussed in detail in another review included in this supplement [58]) differ in complexity, with variable attention given to pain, agitation, and ventilator synchrony. However, it remains to be demonstrated that use of one scale as opposed to any other improves quality of care or patient outcomes. We support the idea that it may be more important that the level of sedation is measured regularly and reproducibly rather than the way it is measured' [59].

To date, no single drug has successfully achieved all of the indications for sedation and analgesia in the ICU. Accordingly, we advocate a combination strategy. Previous recommendations have always necessitated the need for analgesic therapy to ensure that attention is given to pain, and all of the 


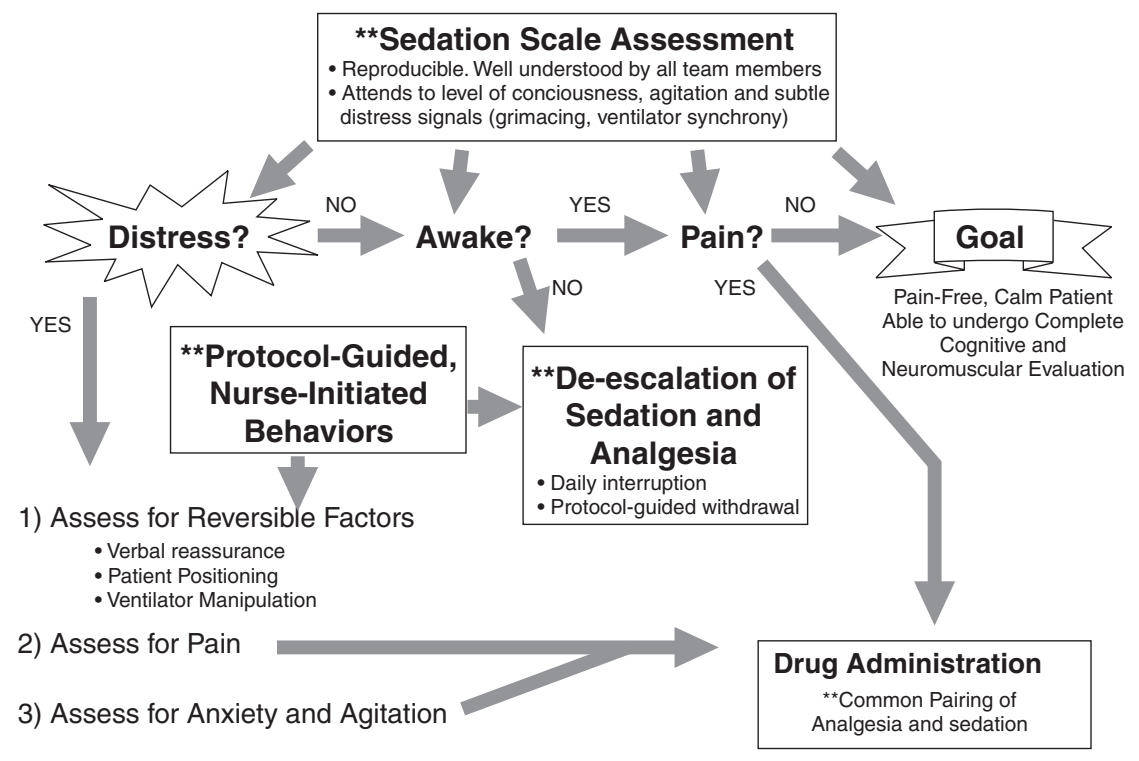

Fundamental components of successful analgesia and sedation strategies. ${ }^{\star \star}$ Denotes components with evidence supporting their benefit.

published, successful sedation protocols incorporate both sedative and analgesic agents. Additionally, new evidence reinforces the perception that combining sedatives and analgesics may confer secondary benefits in achieving optimal sedation. Richman and coworkers [60] conducted a randomized trial of 30 patients with respiratory failure with an expected duration of mechanical ventilation in excess of 48 hours who were receiving a sedative regimen that did not include opiate pain control. Patients were randomly assigned to continuous intravenous sedation with midazolam alone or midazolam plus fentanyl ('co-sedation') during mechanical ventilation, and were evaluated serially using a targeted sedation scale. Compared with the midazolam-only group, the co-sedation group had fewer hours per day with an 'offtarget' Ramsay Sedation Scale score and fewer episodes per day of patient-ventilator asynchrony. Co-sedation was also associated with nonsignificant trends toward a shorter time to achieve sedation, a need for fewer dose titrations per day, and a lower total sedative drug cost, without significant difference between groups in the rate of adverse events.

Two additional comments are worth making. Deep analgesia and sedation with resultant respiratory depression can commonly quell patient-ventilator asynchrony, but optimal management must include manipulation of the ventilator (within the limits of the disease and proven ventilation strategies) to achieve synchrony without relying upon increased drug. This is most commonly achieved by selecting modes that permit more spontaneous than controlled ventilation [61]. Even in the setting of acute lung injury with strict adherence to a low tidal volume ventilation protocol, ventilator manipulation (changes in set respiratory rate, inspiratory flow, and flow profile, with/without volume changes) are feasible and should be entertained before acceleration of drug [62]. Second, although this review highlights the success of uniform practice protocols, consideration to individual patient characteristics should be applied in the selection of drugs to be utilized. Attention to organ system dysfunction may demote certain drugs based on their metabolism (for example, midazolam and morphine metabolites accumulate in patients with renal insufficiency), and a patient history of substance abuse may heighten the appeal of other drugs (benzodiazepines). All successful protocols incorporate limitations in generalizability, and the clinician must recognize when deviation is indicated.

Finally, and most importantly, all of the sedation protocols that have exhibited success have transferred the responsibility for drug manipulation decisions to the bedside nurse. Although a uniform goal should be set through physician and nurse communication at the start of the day (at a minimum), the variable response to drug administration and withdrawal mandates an attendant clinician with the ability to respond in rapid manner - the bedside nurse.

\section{Conclusion}

Sedation is a critical component in the management of the mechanically ventilated patient. Given the poor predictability of drug effect coupled with the hazards of inexact sedation administration, we advocate the implementation of structured assessment tools for pain and depth of sedation, coupled with strategies to guide drug administration and withdrawal. Although de-escalation strategies may differ, protocols that guide the clinician to administer the least necessary sedation 
to achieve patient comfort while maintaining patient-examiner interactivity yield demonstrable improvements in outcome. Although the field needs more large-scale studies to guide the protocol selection process, inattention to this aspect of care may be costly.

\section{Competing interests}

The authors declare that they have no competing interests.

\section{Acknowledgements}

This article is part of Critical Care Volume 12 Supplement 3: Analgesia and sedation in the ICU. The full contents of the supplement are available online at http://ccforum.com/supplements/12/S3.

Publication of the supplement has been funded by an unrestricted grant from GlaxoSmithKline.

\section{References}

1. Puntillo KA: Pain experiences of intensive care unit patients. Heart Lung 1990, 19:526-533.

2. Turner JS, Briggs SJ, Springhorn HE, Potgieter PD: Patients' recollection of intensive care unit experience. Crit Care Med 1990, 18:966-968.

3. Epstein J, Breslow MJ: The stress response of critical illness. Crit Care Clin 1999, 15:17-33, v.

4. Lewis KS, Whipple JK, Michael KA, Quebbeman EJ: Effect of analgesic treatment on the physiological consequences of acute pain. Am J Hosp Pharm 1994, 51:1539-1554.

5. Kollef MH, Levy NT, Ahrens TS, Schaiff R, Prentice D, Sherman G: The use of continuous i.v. sedation is associated with prolongation of mechanical ventilation. Chest 1998, 114:541-548.

6. Ely EW, Inouye SK, Bernard GR, Gordon S, Francis J, May L, Truman B, Speroff T, Gautam S, Margolin R, et al:: Delirium in mechanically ventilated patients: validity and reliability of the confusion assessment method for the intensive care unit (CAM-ICU). JAMA 2001, 286:2703-2710.

7. Ely EW, Margolin R, Francis J, May L, Truman B, Dittus R, Speroff T, Gautam S, Bernard GR, Inouye SK: Evaluation of delirium in critically ill patients: validation of the Confusion Assessment Method for the Intensive Care Unit (CAM-ICU). Crit Care Med 2001, 29:1370-1379.

8. Lin SM, Liu CY, Wang CH, Lin HC, Huang CD, Huang PY, Fang YF, Shieh MH, Kuo HP: The impact of delirium on the survival of mechanically ventilated patients. Crit Care Med 2004, 32: 2254-2259.

9. Ely EW, Shintani A, Truman B, Speroff T, Gordon SM, Harrell FE Jr, Inouye SK, Bernard GR, Dittus RS: Delirium as a predictor of mortality in mechanically ventilated patients in the intensive care unit. JAMA 2004, 291:1753-1762.

10. Pun BT, Ely EW: The importance of diagnosing and managing ICU delirium. Chest 2007, 132:624-636.

11. Thomason JW, Shintani A, Peterson JF, Pun BT, Jackson JC, Ely EW: Intensive care unit delirium is an independent predictor of longer hospital stay: a prospective analysis of 261 non-ventilated patients. Crit Care 2005, 9:R375-R381.

12. Ely EW, Gautam S, Margolin R, Francis J, May L, Speroff T, Truman B, Dittus R, Bernard R, Inouye SK: The impact of delirium in the intensive care unit on hospital length of stay. Intensive Care Med 2001, 27:1892-1900.

13. Pandharipande P, Shintani A, Peterson J, Pun BT, Wilkinson GR, Dittus RS, Bernard GR, Ely EW: Lorazepam is an independent risk factor for transitioning to delirium in intensive care unit patients. Anesthesiology 2006, 104:21-26.

14. Gommers D, Bakker J: Medications for analgesia and sedation in the intensive care unit: an overview. Crit Care 2008, 12 (Suppl 3):S4.

15. Wilhelm W, Kreuer S: The place for short acting opioids: special emphasis on remifentanil. Crit Care 2008, 12(Suppl 3):S5.

16. Chanques G, Jaber S, Barbotte E, Violet S, Sebbane M, Perrigault PF, Mann C, Lefrant JY, Eledjam JJ: Impact of systematic evaluation of pain and agitation in an intensive care unit. Crit Care
Med 2006, 34:1691-1699.

17. Payen JF, Bru O, Bosson JL, Lagrasta A, Novel E, Deschaux I, Lavagne $P$, Jacquot $C$ : Assessing pain in critically ill sedated patients by using a behavioral pain scale. Crit Care Med 2001, 29:2258-2263.

18. Hamill-Ruth RJ, Marohn ML: Evaluation of pain in the critically ill patient. Crit Care Clin 1999, 15:35-54, v-vi.

19. Sessler CN, Gosnell MS, Grap MJ, Brophy GM, O'Neal PV, Keane KA, Tesoro EP, Elswick RK: The Richmond AgitationSedation Scale: validity and reliability in adult intensive care unit patients. Am J Respir Crit Care Med 2002, 166:1338-1344.

20. De Jonghe B, Bastuji-Garin S, Fangio P, Lacherade JC, Jabot J, Appere-De-Vecchi C, Rocha N, Outin H: Sedation algorithm in critically ill patients without acute brain injury. Crit Care Med 2005, 33:120-127.

21. Brattebo G, Hofoss D, Flaatten H, Muri AK, Gjerde S, Plsek PE: Effect of a scoring system and protocol for sedation on duration of patients' need for ventilator support in a surgical intensive care unit. BMJ 2002, 324:1386-1389.

22. MacLaren R, Plamondon JM, Ramsay KB, Rocker GM, Patrick WD, Hall Rl: A prospective evaluation of empiric versus protocol-based sedation and analgesia. Pharmacotherapy 2000, 20: 662-672.

23. Devlin JW, Holbrook AM, Fuller HD: The effect of ICU sedation guidelines and pharmacist interventions on clinical outcomes and drug cost. Ann Pharmacother 1997, 31:689-695.

24. Brook AD, Ahrens TS, Schaiff R, Prentice D, Sherman G, Shannon W, Kollef MH: Effect of a nursing-implemented sedation protocol on the duration of mechanical ventilation. Crit Care Med 1999, 27:2609-2615.

25. Ramsay MA, Savege TM, Simpson BR, Goodwin R: Controlled sedation with alphaxalone-alphadolone. BMJ 1974, 2:656659.

26. Hansen-Flaschen J, Cowen J, Polomano RC: Beyond the Ramsay scale: need for a validated measure of sedating drug efficacy in the intensive care unit. Crit Care Med 1994, 22:732733.

27. Riker RR, Picard JT, Fraser GL: Prospective evaluation of the Sedation-Agitation Scale for adult critically ill patients. Crit Care Med 1999, 27:1325-1329.

28. De Jonghe B, Cook D, Griffith L, Appere-de-Vecchi C, Guyatt G, Theron V, Vagnerre A, Outin $\mathrm{H}$ : Adaptation to the Intensive Care Environment (ATICE): development and validation of a new sedation assessment instrument. Crit Care Med 2003, 31: 2344-2354.

29. Kress JP, Pohlman AS, O'Connor MF, Hall JB: Daily interruption of sedative infusions in critically ill patients undergoing mechanical ventilation. N Engl J Med 2000, 342:1471-1477.

30. Heffner JE: A wake-up call in the intensive care unit. $N$ Engl $J$ Med 2000, 342:1520-1522.

31. Nelson BJ, Weinert CR, Bury CL, Marinelli WA, Gross CR: Intensive care unit drug use and subsequent quality of life in acute lung injury patients. Crit Care Med 2000, 28:3626-3630.

32. Jones C, Griffiths RD, Humphris G, Skirrow PM: Memory, delusions, and the development of acute posttraumatic stress disorder-related symptoms after intensive care. Crit Care Med 2001, 29:573-580.

33. Kress JP, Gehlbach B, Lacy M, Pliskin N, Pohlman AS, Hall JB: The long-term psychological effects of daily sedative interruption on critically ill patients. Am J Respir Crit Care Med 2003, 168:1457-1461.

34. Joseph S: Psychometric evaluation of Horowitz's Impact of Event Scale: a review. J Trauma Stress 2000, 13:101-113.

35. American Psychiatric Association. Anxiety disorders. In Diagnostic and Statistical Manual of Mental Disorders. 4th edition. Washington, DC: American Psychiatric Association; 1994:393-444.

36. Morrow GR, Chiarello RJ, Derogatis LR: A new scale for assessing patients' psychosocial adjustment to medical illness. Psychol Med 1978, 8:605-610.

37. Edwards ND, Alford AM, Dobson PM, Peacock JE, Reilly CS: Myocardial ischaemia during tracheal intubation and extubation. Br J Anaesth 1994, 73:537-539.

38. Srivastava $S$, Chatila W, Amoateng-Adjepong $Y$, Kanagasegar $S$, Jacob B, Zarich S, Manthous CA: Myocardial ischemia and weaning failure in patients with coronary artery disease: an update. Crit Care Med 1999, 27:2109-2112.

39. Chatila W, Ani S, Guaglianone D, Jacob B, Amoateng-Adjepong 
Y, Manthous CA: Cardiac ischemia during weaning from mechanical ventilation. Chest 1996, 109:1577-1583.

40. Conti J, Smith D: Haemodynamic responses to extubation after cardiac surgery with and without continued sedation. $\mathrm{Br}$ $J$ Anaesth 1998, 80:834-836.

41. Hurford WE, Favorito F: Association of myocardial ischemia with failure to wean from mechanical ventilation. Crit Care Med 1995, 23:1475-1480.

42. Kress JP, Vinayak AG, Levitt J, Schweickert WD, Gehlbach BK, Zimmerman F, Pohlman AS, Hall JB: Daily sedative interruption in mechanically ventilated patients at risk for coronary artery disease. Crit Care Med 2007, 35:365-371.

43. Substance Abuse and Mental Health Services Administration: Results from the 2004 National Survey on Drug Use and Health: National Findings. Rockville, MD: US Department of Health and Human Services; 2005.

44. Baldwin WA, Rosenfeld BA, Breslow MJ, Buchman TG, Deutschman CS, Moore RD: Substance abuse-related admissions to adult intensive care. Chest 1993, 103:21-25.

45. Marik $\mathrm{P}$, Mohedin B: Alcohol-related admissions to an inner city hospital intensive care unit. Alcohol Alcohol 1996, 31:393396.

46. Mostafa SM, Murthy BV: Alcohol-associated admissions to an adult intensive care unit: an audit. Eur $J$ Anaesthesiol 2002, 19: 193-196.

47. Moss M, Parsons PE, Steinberg KP, Hudson LD, Guidot DM, Burnham EL, Eaton S, Cotsonis GA: Chronic alcohol abuse is associated with an increased incidence of acute respiratory distress syndrome and severity of multiple organ dysfunction in patients with septic shock. Crit Care Med 2003, 31:869-877.

48. de Wit M, Wan SY, Gill S, Jenvey WI, Best AM, Tomlinson J, Weaver MF: Prevalence and impact of alcohol and other drug use disorders on sedation and mechanical ventilation: a retrospective study. BMC Anesthesiol 2007, 7:3.

49. Fernandez-Sola J, Junque A, Estruch R, Monforte R, Torres A, Urbano-Marquez A: High alcohol intake as a risk and prognostic factor for community-acquired pneumonia. Arch Intern Med 1995, 155:1649-1654.

50. Saitz R, Ghali WA, Moskowitz MA: The impact of alcoholrelated diagnoses on pneumonia outcomes. Arch Intern Med 1997, 157:1446-1452.

51. O'Brien JM Jr, Lu B, Ali NA, Martin GS, Aberegg SK, Marsh CB, Lemeshow S, Douglas IS: Alcohol dependence is independently associated with sepsis, septic shock, and hospital mortality among adult intensive care unit patients. Crit Care Med 2007, 35:345-350.

52. Moss M, Bucher B, Moore FA, Moore EE, Parsons PE: The role of chronic alcohol abuse in the development of acute respiratory distress syndrome in adults. JAMA 1996, 275:50-54.

53. Jacobi J, Fraser GL, Coursin DB, Riker RR, Fontaine D, Wittbrodt ET, Chalfin DB, Masica MF, Bjerke HS, Coplin WM, et al.: Clinical practice guidelines for the sustained use of sedatives and analgesics in the critically ill adult. Crit Care Med 2002, 30: 119-141.

54. Carson SS, Kress JP, Rodgers JE, Vinayak A, Campbell-Bright $S$, Levitt J, Bourdet S, Ivanova A, Henderson AG, Pohlman A, et al:: A randomized trial of intermittent lorazepam versus propofol with daily interruption in mechanically ventilated patients. Crit Care Med 2006, 34:1326-1332.

55. Mehta S, Burry L, Fischer S, Martinez-Motta JC, Hallett D, Bowman D, Wong C, Meade MO, Stewart TE, Cook DJ: Canadian survey of the use of sedatives, analgesics, and neuromuscular blocking agents in critically ill patients. Crit Care Med 2006, 34:374-380.

56. Rhoney DH, Murry KR: National survey of the use of sedating drugs, neuromuscular blocking agents, and reversal agents in the intensive care unit. J Intensive Care Med 2003, 18:139145.

57. Egerod I, Christensen BV, Johansen L: Trends in sedation practices in Danish intensive care units in 2003: a national survey. Intensive Care Med 2006, 32:60-66.

58. Sessler CN, Grap MJ, Ramsay MAE: Evaluating and monitoring analgesia and sedation in the intensive care unit. Crit Care 2008, 12(Suppl 3):S2.

59. Deem S: Monitoring delirium and sedation. Crit Care Med 2006, 34:2264-2265.

60. Richman PS, Baram D, Varela M, Glass PS: Sedation during mechanical ventilation: a trial of benzodiazepine and opiate in combination. Crit Care Med 2006, 34:1395-1401.

61. Rathgeber J, Schorn B, Falk V, Kazmaier S, Spiegel T, Burchardi $\mathrm{H}$ : The influence of controlled mandatory ventilation (CMV), intermittent mandatory ventilation (IMV) and biphasic intermittent positive airway pressure (BIPAP) on duration of intubation and consumption of analgesics and sedatives. A prospective analysis in $\mathbf{5 9 6}$ patients following adult cardiac surgery. Eur J Anaesthesiol 1997, 14:576-582.

62. The Acute Respiratory Distress Syndrome Network: Ventilation with lower tidal volumes as compared with traditional tidal volumes for acute lung injury and the acute respiratory distress syndrome. N Engl J Med 2000, 342:1301-1308.
Disclaime

This article is part of Critical Care Volume 12 Supplement 3: Analgesia and sedation in the ICU. Publication of the supplement has been funded by an unrestricted grant from GlaxoSmithKline. GlaxoSmithKline has had no editorial control in respect of the articles contained in this publication.

The opinions and views expressed in this publication are those of the authors and do not constitute the opinions or recommendations of the publisher or GlaxoSmithKline. Dosages, indications and methods of use for medicinal products referred to in this publication by the authors may reflect their research or clinical experience, or may be derived from professional literature or other sources. Such dosages, indications and methods of use may not reflect the prescribing information for such medicinal products and are not recommended by the publisher or GlaxoSmithKline. Prescribers should consult the prescribing information approved for use in their country before the prescription of any medicinal product. Whilst every effort is made by the publisher and editorial board to see that no inaccurate or misleading data, opinion, or statement appear in this publication, they wish to make it clear that the data and opinions appearing in the articles herein are the sole responsibility of the that the data and opinions
contributor concerned.

Accordingly, the publishers, the editor and editorial board, GlaxoSmithKline, and their respective employees, officers and agents accept no liability whatsoever for the consequences of such inaccurate or misleading data, opinion or statement. 